Supporting Information for

\title{
Sirtuins 1 and 2 are Universal Histone Deacetylases
}

Willie W. Hsu, Bo Wu, and Wenshe R. Liu*

Department of Chemistry, Texas A\&M University, College Station, TX 77843 


\section{HisTag and Cleavage Site Protein Sequence}

G S S H H H H H H S Q D P E N L Y F Q

\section{Histone H2A Protein Sequence}

S G R G K Q G G K A R A K A K T R S S R A G L Q F P V G R V H R L L R K G N Y A E R V GA G A PVYLAAVLEYLTA E I LELAGNAARDNKKT R I P R H L Q LA IR N D E E L N K L L G KV T I A Q G GVLPN I Q A V LLPKK T E S H H KAKG K

\section{Histone H2B Protein Sequence}

S E P A K S A P A P K K GS K KA V T KA Q K K D G K KR K R S R K E S Y S VYVYKV L K QVH P D T GISS KA M G I M N S F V D IF ERIAG E A S R L H Y N KR S T I T S R E I Q T AVR L L L P G E L A K H AVS E G T K A V T KY T S S K

\section{Histone H3 Protein Sequence}

A R T K Q T A R K S T G GK A P R K Q L A T KA A R K S A P A T G G VK K P H R Y R P G TVALREIRRYQKSTELLIRKLPF QRLVREIAQ D F K T D L R F QS S A V M A L Q E A S E A L V G L F E D T N L A A I H A KRVT I M P K D I Q L A R R IR G E R A

\section{Histone H4 Protein Sequence}

S G R G K G G K G L G K G G A K R H R K V L R D N I Q G I T K P A I R R L A R R G G VK R IS G L IY E E T R G V L K V F L E N VIR D A V TYT E H A KR K T V T A M D VVY A L KR Q GRT L Y G F G G

\section{HisTag-SUMO Protein Sequence}

M G S S H H H H H H S S G L V P R G S H M S D S E V N Q E A K P E V K P E V K P E T H IN LKVS D GSSE IFFKIKK T T P LRRL M EAFAKR Q GKE M D S R F L Y D GIR I Q A D Q T P E D L D M E D N D I E A H RE Q I GG

\section{HisTag and Cleavage Site DNA Sequence}

catcaccatcatcaccacagccaggatccggaaaatctgtacttccag

\section{Histone H2A DNA Sequence}

tctggtcgtggtaaacaaggtggtaaagcacgtgcaaaggctaagactcgtagcagccgtgccggtctgcagtttccagt gggtcgcgttcaccgtctgctgcgtaaaggcaactatgctgaacgtgtgggtgctggtgcaccggtttacctggcagctgt actggaatatctgaccgcagagattctggagctggcaggtaacgcagctcgtgataataagaagacccgcatcatcccac gtcacctgcagctggccatccgcaacgatgaggaactgaacaaactgctgggcaaagttactatcgctcaaggtggcgtt ctgccgaacatccaggcagttctgctgccgaagaaaaccgaatcccaccacaaagcgaaaggtaagtga

\section{Histone H2B DNA Sequence}

tcagaaccagctaagtctgcaccggctccgaagaaaggctctaagaaggctgttaccaaggctcagaagaaagatggta agaaacgcaaacgttctcgtaaagaaagctattctgtgtacgtgtataaagttctgaaacaagtacatccagacactggc atttccagcaaagcgatgggcattatgaacagcttcgttaacgatatcttcgaacgtatcgcaggcgaagcgagccgtctg gctcactataacaaacgttctaccatcacctctcgtgaaattcaaactgcagttcgtctgctgctgccaggtgaactggcta aacacgcggttagcgaaggcactaaagcagttaccaaatacacttcttccaaatga 


\section{Histone H3 DNA Sequence}

gctcgcaccaaacagactgctcgtaagtccactggcggtaaagcgccgcgtaaacagctggcaaccaaggcagcgcgt

aaaagcgctccagctactggcggcgtgaagaagccgcaccgttatcgcc gggtactgtggctctgcgtgaaatccgccg ctaccagaaaagcaccgaactgctgattcgcaaactgccatttcaacgtctggttcgcgaaattgctcaggatttcaaaac cgacctgcgcttccagtctagcgctgtgatggcactgcaagaggcgtctgaggcatatctggttggcctgttcgaagatac caacctgtgcgcaatccatgcaaagcgtgtaaccattatgccgaaagacatccaactggctcgtcgtatccgtggtgagc gtgcgtga

\section{Histone H4 DNA Sequence}

tctggtcgtggtaaaggtggtaaaggcctgggtaaaggtggtgctaagcgtcaccgtaaagtgctgcgcgacaacatcca gggtatcaccaaaccagctattcgccgtctggcacgtcgcggtggtgtgaaacgcatcagcggtctgatctatgaagaaa cccgtggtgttctgaaagtatttctggagaacgttatccgcgatgcggtgacctacaccgaacacgcgaaacgtaagacc gttactgctatggatgttgtgtacgctctgaaacgccagggtcgtactctgtacggtttcggtggctga

\section{HisTag-SUMO DNA Sequence}

Atgggcagcagccatcatcatcatcatcacagcagcggcctggtgccgcgcggcagccatatgtcggactcagaagtca atcaagaagctaagccagaggtcaagccagaagtcaagcctgagactcacatcaatttaaaggtgtccgatggatcttca gagatcttcttcaagatcaaaaagaccactcctttaagaaggctgatggaagcgttcgctaaaagacagggtaaggaaat ggactccttaagattcttgtacgacggtattagaattcaagctgatcagacccctgaagatttggacatggaggataacga tattattgaggctcacagagaacagattggtggt

\section{Sirtuin 1 and Sirtuin 2 Acetyl-nucleosome Assay Evaluation}

The raw data obtained from the assay were normalized using the following equation $y=\frac{x_{i}-x_{n c}}{x_{p c}-x_{n c}} x 100$, where $x_{i}$ was the RLU value of interest, $x_{n c}$ was the RLU value of the negative control (wild-type nucleosome), and $x_{p c}$ was the RLU value of the positive control (acetyl-nucleosome). 


\section{Supplementary Figures}

Comparison of Acetyl-nucleosome Signal with Wild-type Nucleosome

RLU Signal Comparison Between

Wild-type Nucleosome and Acetyl-nucleosome

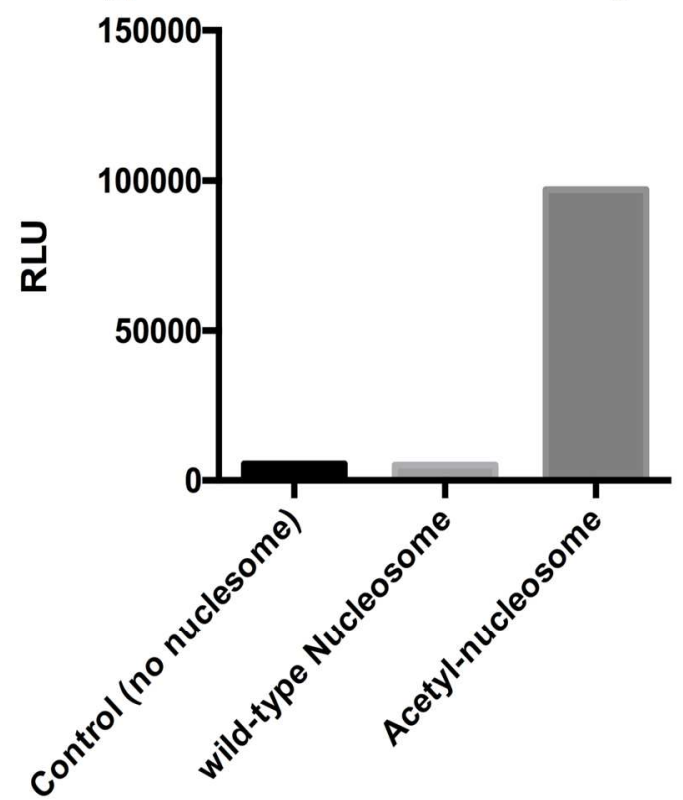

Supplementary Figure 1. Comparative RLU signal between wild-type nucleosome and an acetyl-nucleosome. 